\title{
RESPUESTA INFLAMATORIA POSTOPERATORIA EN PACIENTES CON CÁNCER GÁSTRICO RESECADO*
}

\author{
Drs. Marcelo A. Beltrán S. ${ }^{1,2}$, Javier Arcos P. ${ }^{2}$, Oriana Urbina C. ${ }^{1,2}$, \\ Francisco Rodríguez V. ${ }^{2}$, Ramón Hermosilla V. ${ }^{2}$, Tatiana Fuentes M. ${ }^{1,2}$ \\ 1 Servicio de Cirugía, Hospital de La Serena. \\ 2 Departamento de Clínicas, Facultad de Medicina, Universidad Católica del Norte, La Serena. \\ Chile.
}

\begin{abstract}
Postoperative inflammatory response in patients with gastric cancer submitted to total and partial distal gastrectomy
\end{abstract}

Introduction: The removal of the stomach causes an acute inflammatory response. In some cases this response is exaggerated due to complications. The main purpose of this study was to describe the expected inflammatory response to gastrectomy. Methods: This is a prospective analysis of consecutive patients submitted to gastrectomy for gastric cancer. For analytical purposes, the cohort was divided in complicated and uncomplicated patients. Uncomplicated patients were further divided in total gastrectomy and partial gastrectomy. The main outcome measure was to compare the inflammatory response of uncomplicated and complicated patients. The secondary outcome measure was to compare the inflammatory response of uncomplicated patients submitted to total and partial gastrectomy along the timeline. Results: Preoperative values were similar between groups. At $24 \mathrm{~h}$ after gastrectomy, complicated patients showed significantly higher values for D-dimer, CRP and WBC. Further analysis comparing uncomplicated patients showed normal preoperative values and a mild elevation of inflammatory parameters which was similar in both groups of patients. Conclusions: The expected systemic inflammatory response to partial or total gastrectomy was mild. Elevations of inflammatory values were shown early by complicated patients, constituting reliable parameters to identify postoperative inflammatory complications.

Key words: Gastric cancer, gastrectomy, inflammatory response, C-reactive protein, D-Dimer.

\section{Resumen}

Introducción: La extirpación del estómago produce una respuesta inflamatoria aguda. En algunos casos esta respuesta es exagerada debido al desarrollo de complicaciones. El objetivo general del presente estudio es el de describir la respuesta inflamatoria esperable en pacientes sometidos a gastrectomía.

\footnotetext{
*Recibido el 11 de octubre de 2014 y aceptado para publicación el 2 de diciembre de 2014.

Conflicto de intereses: Ninguno.

Correspondencia: Marcelo A. Beltrán S. beltran_01@yahoo.com
} 
Métodos: Análisis prospectivo de pacientes consecutivos sometidos a gastrectomía por cáncer gástrico. La cohorte se analizó dividida en pacientes sin complicaciones y pacientes que sufrieron complicaciones. Los pacientes sin complicaciones fueron además divididos en pacientes sometidos a gastrectomía total y parcial. El objetivo principal fue comparar la respuesta inflamatoria de pacientes complicados con pacientes sin complicaciones. El objetivo secundario fue comparar la respuesta inflamatoria de los pacientes no complicados sometidos a gastrectomía total con los pacientes sometidos a gastrectomía parcial. Resultados: Los valores de los exámenes preoperatorios fueron similares entre todos los grupos. A las $24 \mathrm{~h}$ después de la gastrectomía, los pacientes complicados mostraron valores significativamente mayores de la proteína $\mathrm{C}$ reactiva, leucocitos y dímero-D. El análisis comparativo de los pacientes sin complicaciones, sometidos a gastrectomía total y parcial, mostró una moderada elevación de los parámetros inflamatorios, la cual fue similar en ambos grupos. Conclusiones: La respuesta inflamatoria esperable a la gastrectomía total o parcial es moderada. Los pacientes que sufrieron complicaciones mostraron precozmente una importante elevación de sus parámetros inflamatorios lo que permite sospechar el desarrollo de complicaciones postoperatorias.

Palabras clave: Cáncer gástrico, gastrectomía, respuesta inflamatoria, proteína $\mathrm{C}$ reactiva, Dímero-D.

\section{Introducción}

La relación íntima que existe entre el desarrollo y progresión del cáncer y la respuesta inflamatoria del organismo humano se conoce desde el siglo XIX ${ }^{1}$. La inflamación causada por el cáncer es modulada por las células cancerígenas, las células del huésped y las interacciones entre ellas. El crecimiento del cáncer y la consecuente invasión producen daño tisular local, alterando la homeostasis, lo que lleva al desarrollo de una respuesta sistémica de fase aguda $^{1,2}$. Por estas razones, los pacientes portadores de cáncer en el estómago o en cualquier otra localización anatómica, presentan algún grado de inflamación sistémica. Marcadores inflamatorios séricos de fase aguda como la proteína $\mathrm{C}$ reactiva (PCR) y los glóbulos blancos (GB), además de marcadores de la coagulación como el dímero-D (DD) y parámetros nutricionales relacionados como la albúmina, han sido utilizados para evidenciar esta respuesta sistémica al cáncer y basándose en la intensidad de la respuesta predecir la presencia de enfermedad avanzada y el pronóstico ${ }^{1-8}$. La gastrectomía constituye el principal tratamiento curativo o paliativo para pacientes con cáncer gástrico. Así como con cualquier otro procedimiento quirúrgico, la remoción del estómago causa una respuesta inflamatoria en el organismo humano, la cual se suma a la respuesta inflamatoria propia del cáncer. Consecuentemente, la presencia de respuesta inflamatoria a la gastrectomía es esperable y está presente en todos los pacientes operados sin complicaciones. En algunos casos, esta respuesta es exagerada debido a complicaciones propias del procedimiento ${ }^{9,10}$. El objetivo del presente estudio es el de evidenciar y describir la respuesta inflamatoria esperable del organismo humano a la gastrectomía en pacientes con cáncer gástrico.

\section{Material y Método}

\section{Diseño}

En el Servicio de Cirugía del Hospital San Juan de Dios de La Serena, desde el año 2006 se mantiene una base de datos prospectiva para pacientes operados por cáncer gástrico en la cual se ingresan rutinariamente diferentes variables entre las que se encuentran algunos parámetros inflamatorios tales como la PCR y GB, parámetros de la coagulación como el DD, además de la albúmina sérica. Estos parámetros se miden en el período preoperatorio inmediato y después de la cirugía a las $24 \mathrm{~h}, 48 \mathrm{~h}$ y 5 días. Utilizando esta base de datos, se diseñó un estudio prospectivo comparativo para evidenciar la respuesta inflamatoria a la gastrectomía en una cohorte de pacientes seleccionados sometidos a gastrectomía con disección linfonodal D2 por adenocarcinoma gástrico entre los meses de enero del año 2006 hasta agosto del año 2014 (Figura 1).

\section{Definiciones y valores normales}

El estadio II se definió de acuerdo a la clasificación TNM del American Joint Committee on Cancer (AJCC) ${ }^{11}$. En el estudio se incluyeron todos los pacientes con estadio II sin diferenciarlos por la subdivisión IIA y IIB. En nuestra institución, son valores normales para DD $<200 \mathrm{ng} / \mathrm{mL}, \mathrm{PCR}$ de 0 a $5 \mathrm{mg} / \mathrm{L}, \mathrm{GB}$ entre 4.400 y $11.300 \mathrm{x} \mathrm{mm}^{3}$ y para albúmina 3,4 a 4,8 g/dL. El denominado Glasgow Prognostic Score (GPS) fue descrito el año 2003 por Forrest et $\mathrm{al}^{12,13}$, subsecuentemente fue utilizado en la evaluación preoperatoria de pacientes con cáncer gástrico como predictor de enfermedad avanzada ${ }^{1-4}$. Pacientes con PCR elevada e hipoalbuminemia asociada tienen un puntaje de 2. Pacientes con uno solo de estos parámetros bioquímicos alterado se les asigna un puntaje de 1 . Pacientes con ninguno 


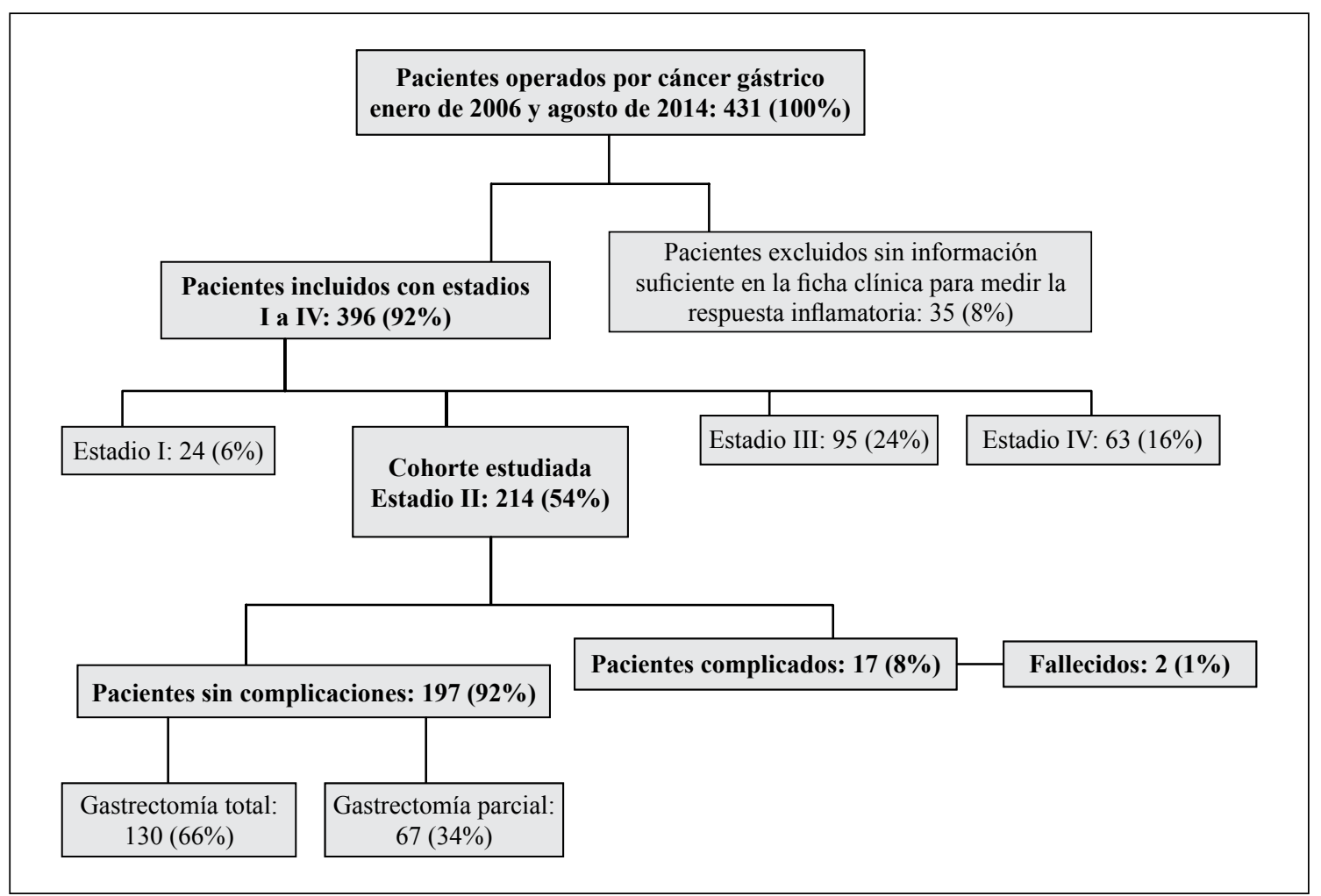

Figura 1. Flujograma de inclusión de pacientes en el estudio.

de estos parámetros alterado tienen un puntaje de $0^{4,12,13}$. Las complicaciones postoperatorias son reportadas de acuerdo a la clasificación de Clavien ${ }^{14}$. Se definió como paciente con patología pulmonar a todo paciente con criterios de enfermedad bronquial obstructiva crónica de cualquier etiología o pacientes con criterios de insuficiencia respiratoria. Se definió como pacientes con patología renal a todo paciente con criterios de insuficiencia renal crónica en cualquiera de sus estadios.

\section{Criterios de inclusión}

Fueron incluidos pacientes con cáncer gástrico estadio preoperatorio II confirmado por estudio histopatológico con un GPS de 1 o menor. Se incluyeron pacientes en estadio II debido a que constituyen el mayor grupo de pacientes operados en nuestra institución dentro del período de estudio y debido también a que por su estadio bajo todos ellos tenían un GPS de 1 o menor, ajustándose por lo tanto, a los criterios de inclusión y contribuyendo a la uniformidad de la muestra.

\section{Grupos de estudio}

La cohorte fue dividida en pacientes que sufrieron complicaciones en el postoperatorio y pacientes que no tuvieron complicaciones. Los pacientes sin complicaciones fueron además divididos en pacientes operados mediante gastrectomía total y gastrectomía parcial distal la que incluye la antrectomía, hemigastrectomía y gastrectomía subtotal (Figura 1).

\section{Objetivos de respuesta}

El objetivo principal fue la comparación de la respuesta inflamatoria en pacientes con y sin complicaciones postoperatorias después de la gastrectomía a las 24 h, 48 h y 5 días. El objetivo secundario fue la comparación de la respuesta inflamatoria esperable de los pacientes sin complicaciones sometidos a gastrectomía total y parcial medida a las $24 \mathrm{~h}, 48 \mathrm{~h}$ y 5 días después de la cirugía.

\section{Estadística}

El análisis estadístico fue realizado con el programa SPSS versión 11.0 (Chicago, Illinois, USA). Las variables categóricas dicotómicas se reportaron como proporciones, las variables continuas se reportaron como valores promedio con desviación estándar. El análisis comparativo se realizó con el test exacto de Fisher y el test de Mann-Whitney según fuera apropiado. 


\section{Resultados}

Se estudió una cohorte de 214 pacientes $(100 \%)$ con cáncer gástrico en estadio II. De ellos, 197 pacientes $(92 \%)$ tuvieron un curso postoperatorio sin complicaciones. En 17 casos $(8 \%)$ se produjeron complicaciones, falleciendo 2 pacientes $(1 \%)$ (Figura 1).

\section{Comparación entre pacientes complicados y pacientes sin complicaciones}

La mayoría de los pacientes en ambos grupos fueron hombres con edad, índice de masa corporal
(IMC) y hábitos como tabaquismo y consumo de alcohol similares. Las comorbilidades fueron en general comparables pero los pacientes sin complicaciones tenían una mayor incidencia de enfermedades cardiológicas y patología pulmonar crónica que los pacientes con complicaciones (Tabla 1). En la Tabla 2 se muestra la evolución del DD, PCR, GB y albúmina desde los valores basales del preoperatorios hasta el $5^{\circ}$ día después de la cirugía. Los valores preoperatorios fueron similares entre los dos grupos. Después de la gastrectomía, a las $24 \mathrm{~h}$ los pacientes que desarrollaron complicaciones mostraron valores significativamente elevados de los marcadores infla-

Tabla 1. Características generales de 214 pacientes con cáncer gástrico en estadio II

\begin{tabular}{|lcccc|}
\hline Pacientes & Sin complicaciones: 197 (92\%) & Complicaciones: 17 (8\%) & Total: $\mathbf{2 1 4}(\mathbf{1 0 0} \%)$ & p \\
Sexo: M/F & $126(64 \%) / 71(36 \%)$ & $9(53 \%) / 8(47 \%)$ & $135(63 \%) / 79(37 \%)$ & 0,223 \\
Edad & $64 \pm 15(25-88)$ & $67 \pm 10(48-75)$ & $64 \pm 14(25-88)$ & 0,436 \\
IMC & $23 \pm 2(18-29)$ & $23 \pm 3(19-30)$ & $23 \pm 2(18-30)$ & 0,674 \\
Alcoholismo & $78(39 \%)$ & $6(35 \%)$ & $84(39 \%)$ & 0,525 \\
Tabaquismo & $104(53 \%)$ & $10(58 \%)$ & $114(53 \%)$ & 0,201 \\
Diabetes & $15(8 \%)$ & $2(11 \%)$ & $17(8 \%)$ & 0,287 \\
Hipertensión arterial & $74(37 \%)$ & $8(47 \%)$ & $(38 \%)$ & 0,036 \\
Enfermedad coronaria & $4(2 \%)$ & - & $4(2 \%)$ & 0,176 \\
Patología pulmonar & $57(29 \%)$ & $1(6 \%)$ & $58(27 \%)$ & 0,001 \\
Enfermedad renal & $2(1 \%)$ & - & $2(1 \%)$ & 0,138 \\
\hline
\end{tabular}

Tabla 2. Comparación de la respuesta inflamatoria postoperatoria entre los pacientes con y sin complicaciones

\begin{tabular}{|lccr|}
\hline Mediciones & Sin complicaciones & Complicaciones & p \\
Dímero-D (ng/mL) & & & 0,322 \\
Preoperatorio & $56 \pm 41,2(10-263)$ & $48,6 \pm 14,3(30-70)$ & 0,003 \\
Postoperatorio 24 h & $393,6 \pm 358,3(85-1.920)$ & $841,4 \pm 486,1(380-1.720)$ & 0,006 \\
Postoperatorio 48 h & $688 \pm 639(100-2.340)$ & $1354,3 \pm 478,8(890-2.180)$ & $<0,0001$ \\
Postoperatorio 5 días & $508 \pm 434,3(30-1.850)$ & $2084,3 \pm 238,4(1.760-2.450)$ & \\
PCR (mg/L) & & & 0,386 \\
Preoperatorio & $3,1 \pm 1,7(0,2-6)$ & $3,7 \pm 1,8(0,3-6)$ & 0,018 \\
Postoperatorio 24 h & $136 \pm 61,5(40-330)$ & $245,7 \pm 76,5(190-360)$ & $<0,0001$ \\
Postoperatorio 48 h & $127 \pm 47,5(30-210)$ & $297 \pm 43,5(230-470)$ & $<, 0001$ \\
Postoperatorio 5 días & $59 \pm 28,8(25-150)$ & $328,6 \pm 56,6(290-480)$ & 0,850 \\
Albúmina (g/dL) & $4 \pm 0,5(3,6-5,1)$ & & 0,046 \\
Preoperatorio & $2,8 \pm 0,5(1,5-3,8)$ & $4 \pm 0,4(3,6-4,8)$ & $<0,0001$ \\
Postoperatorio 24 h & $2,8 \pm 0,5(1,6-3,8)$ & $2,3 \pm 0,4(1,9-2,8)$ & 0,009 \\
Postoperatorio 48 h & $3,1 \pm 0,5(1,8-3,9)$ & $1,9 \pm 0,2(1,6-2,3)$ & \\
Postoperatorio 5 días & & $2,5 \pm 0,5(1,6-3)$ & 0,336 \\
GB (x mm $\left.{ }^{3}\right)$ & & & 0,865 \\
Preoperatorio & $6.098 \pm 1.245(4.100-8.800)$ & $6.586 \pm 1.476(5.200-8.700)$ & 0,006 \\
Postoperatorio 24 h & $15.039 \pm 2.614(10.000-19.500)$ & $15.214 \pm 2.177(12.500-18.000)$ & $<0,0001$ \\
Postoperatorio 48 h & $11.995 \pm 2.920(6.000-17.500)$ & $15.185 \pm 1.847(12.000-17.500)$ & $13.157 \pm 1.919(10500-16.400)$ \\
Postoperatorio 5 días & $8.480 \pm 1.549(5.200-13.500)$ & $13.150)$ & \\
\hline
\end{tabular}


matorios comparados con los pacientes sin complicaciones y un descenso progresivo de la albúmina, la cual mostró una leve recuperación al $5^{\circ}$ día. Las complicaciones más frecuentes fueron respiratorias e infecciosas. Las complicaciones más severas fueron las relacionadas con las filtraciones de la anastomosis, en dos casos los pacientes fallecieron (Tabla 3).

\section{Comparación entre pacientes sometidos a gastrectomía total y parcial}

El análisis de los pacientes que no sufrieron complicaciones, comparando pacientes sometidos a gastrectomía total y parcial, muestra que los pacientes sometidos a gastrectomía parcial son de mayor edad comparados con los pacientes sometidos a gastrectomía total. Respecto al IMC, hábitos y comorbilidades, ambos grupos fueron similares (Tabla 4). En la Tabla 5 se muestra la evolución de los parámetros inflamatorios y de la albúmina desde el preoperatorio hasta el $5^{\circ}$ día después de la cirugía. Los valores preoperatorios fueron normales en todos los pacientes y se alteraron moderadamente y dentro de los esperado durante las primeras 24 a 48 h después de la cirugía, retornando prácticamente hasta sus valores basales durante los siguientes días como se evidencia por las mediciones efectuadas al $5^{\circ}$ día postoperatorio.

\section{Discusión}

El cáncer gástrico induce una respuesta inflamatoria sistémica crónica moderada. Se ha demostrado que los pacientes con enfermedad avanzada sufren una respuesta inflamatoria más severa. Basándose en esta respuesta, se han utilizado
Tabla 3. Complicaciones en 17 pacientes

\begin{tabular}{|lcc|}
\hline Complicación & $\begin{array}{c}\text { Pacientes } \\
\mathbf{1 7}(\mathbf{1 0 0} \%)\end{array}$ & $\begin{array}{c}\text { Grado de } \\
\text { Clavien }\end{array}$ \\
\hline Atelectasias & $9(52 \%)$ & I \\
$\begin{array}{l}\text { Infección de sitio quirúrgico } \\
\text { superficial }\end{array}$ & $7(41 \%)$ & I \\
\hline Neumonía & $6(35 \%)$ & II \\
Filtración de la anastomosis & $4(23 \%)$ & IIIb \\
Absceso intraperitoneal & $2(12 \%)$ & IVa \\
\hline Choque séptico & $2(12 \%)$ & IVb \\
\hline Mortalidad & $2(12 \%)$ & - \\
\hline
\end{tabular}

* Representa $1 \%$ de la mortalidad general de la cohorte.

marcadores de respuesta inflamatoria como la PCR como factores pronósticos de enfermedad avanza$\mathrm{da}^{1-4}$. Así también, la hipoalbuminemia asociada a la respuesta inflamatoria se ha relacionado a pacientes con cáncer gástrico avanzado ${ }^{2}$. Finalmente, algunos parámetros de la coagulación, principalmente el DD, han demostrado su valor pronóstico en pacientes con cáncer gástrico avanzado ${ }^{5-8}$. Estos marcadores inflamatorios, nutricionales y hemostáticos utilizados como factores pronósticos en pacientes con cáncer gástrico son los mismos parámetros que se observan alterados en la respuesta inflamatoria sistémica esperable después de una gastrectomía y también como respuesta inflamatoria de fase aguda a complicaciones secundarias a gastrectomía por cáncer gástrico ${ }^{9,10}$. Aun cuando esta respuesta inflamatoria a las complicaciones ha sido estudiada, no se encuentran publicaciones que describan la respuesta

Tabla 4. Características generales de 197 pacientes sin complicaciones

\begin{tabular}{|lcccc|}
\hline & $\begin{array}{c}\text { Gastrectomía total } \\
\mathbf{1 3 0}(\mathbf{6 6 \% )}\end{array}$ & $\begin{array}{c}\text { Gastrectomía parcial } \\
\mathbf{6 7}(\mathbf{3 4 \% )}\end{array}$ & $\begin{array}{c}\text { Total } \\
\mathbf{1 9 7}(\mathbf{1 0 0} \%)\end{array}$ & $\mathbf{p}$ \\
\hline Sexo: M/F & $77(59 \%) / 53(41 \%)$ & $49(73 \%) / 18(27 \%)$ & $126(64 \%) / 71(36 \%)$ & 0,002 \\
Edad & $62 \pm 16,3(25-88)$ & $68 \pm 10,5(43-83)$ & $64 \pm 15(25-88)$ & 0,089 \\
IMC & $21 \pm 2(18-26)$ & $23 \pm 2(19-29)$ & $23 \pm 2(18-29)$ & 0,426 \\
Alcoholismo & $49(38 \%)$ & $29(43 \%)$ & $78(39 \%)$ & 0,128 \\
Tabaquismo & $70(54 \%)$ & $34(51 \%)$ & $104(53 \%)$ & 0,814 \\
Diabetes & $10(8 \%)$ & $5(7 \%)$ & $15(8 \%)$ & 0,836 \\
Hipertensión arterial & $49(38 \%)$ & $25(37 \%)$ & $74(37 \%)$ & 0,851 \\
Enfermedad coronaria & $1(1 \%)$ & $3(4 \%)$ & $4(2 \%)$ & 0,277 \\
Patología pulmonar & $38(29 \%)$ & $19(28 \%)$ & $57(29 \%)$ & 0,827 \\
Enfermedad renal & $2(1 \%)$ & - & $2(1 \%)$ & 0,114 \\
\hline
\end{tabular}


Tabla 5. Comparación de la respuesta inflamatoria postoperatoria entre pacientes sin complicaciones sometidos a gastrectomía total y parcial

\begin{tabular}{|lccc|}
\hline Mediciones & Gastrectomía total & Gastrectomía parcial & p \\
Dímero-D (ng/mL) & & & 0,004 \\
Preoperatorio & $43,7 \pm 27,7(10-90)$ & $79,7 \pm 52,1(50-263)$ & 0,961 \\
Postoperatorio 24 h & $391,8 \pm 294,6(90-1.520)$ & $464,2 \pm 391,1(85-1.920)$ & 0,869 \\
Postoperatorio 48 h & $656,9 \pm 649,7(105-2.250)$ & $761,3 \pm 618,6(100-2.340)$ & 0,809 \\
Postoperatorio 5 días & $454,5 \pm 422,3(30-1.450)$ & $607,7 \pm 457,4(55-1.809)$ & 0,310 \\
PCR (mg/L) & & & 0,690 \\
Preoperatorio & $3,3 \pm 1,7(0,3-6)$ & $2,8 \pm 1,9(0,2-5)$ & 0,958 \\
Postoperatorio 24 h & $131,8 \pm 78,2(40,7-323)$ & $144 \pm 59,8(40-330)$ & 0,653 \\
Postoperatorio 48 h & $117,5 \pm 70,6(30-205)$ & $125,9 \pm 71,8(45-210)$ & \\
Postoperatorio 5 días & $66,4 \pm 22,8(35-128,2)$ & $78 \pm 72,1(25-150)$ & 0,536 \\
Albúmina (g/dL) & $4 \pm 0,4(3,6-4,8)$ & $4,1 \pm 0,5(3,8-5,1)$ & 0,397 \\
Preoperatorio & $2,7 \pm 0,5(1,5-3,6)$ & $2,8 \pm 0,5(1,7-3,8)$ & 0,317 \\
Postoperatorio 24 h & $2,8 \pm 0,5(1,6-3,6)$ & $2,9 \pm 0,5(1,8-3,8)$ & 0,509 \\
Postoperatorio 48 h & $3 \pm 0,5(1,8-3,8)$ & $3,1 \pm 0,5(2-3,9)$ & $<0,0001$ \\
Postoperatorio 5 días & & & 0,912 \\
GB (x mm $\left.{ }^{3}\right)$ & $6.477 \pm 1.323(5.200-8.800)$ & $5.373 \pm 623(4.100-5.900)$ & 0,307 \\
Preoperatorio & $15.065 \pm 2.594(10.000-19.500)$ & $14.989 \pm 2.709(10.200-19.000)$ & 0,390 \\
Postoperatorio 24 h & $12.268 \pm 2.845(6.000-17.500)$ & $11.473 \pm 3051(6.500-17.000)$ & \\
Postoperatorio 48 h & $8.604 \pm 1.482(5.200-13.500)$ & $8.243 \pm 1.678(5.300-12.200)$ & \\
Postoperatorio 5 días & & & \\
\hline
\end{tabular}

inflamatoria sistémica esperable después de una gastrectomía sin complicaciones en pacientes con cáncer gástrico. En el presente reporte, se seleccionó una cohorte específica de pacientes para estudiar la respuesta inflamatoria normal a la gastrectomía. Se incluyeron pacientes con un GPS menor a 1 con la intención de disminuir la posibilidad de sesgo incluyendo pacientes con enfermedad avanzada en quienes se esperan valores anormales de PCR, DD y albúmina. De todos los pacientes con cáncer gástrico sometidos a gastrectomía, el grupo que se ajusta mejor a este criterio es el de los pacientes en estadio II. La respuesta inflamatoria se objetivó mediante el uso de marcadores inflamatorios como los GB y la PCR, además de la albúmina que actúa como un marcador inflamatorio debido a su asociación con las alteraciones de la PCR; y el DD que sufre alteraciones cuando el organismo sufre lesiones vasculares asociándose por lo tanto, a la respuesta inflamatoria.

\section{Pacientes complicados versus pacientes sin complicaciones}

A las $24 \mathrm{~h}$ después de la cirugía, aquellos pacientes que sufrieron complicaciones tenían los valores de los parámetros inflamatorios medidos marcadamente alterados comparados con los pacientes que tuvieron un curso postoperatorio sin complicaciones. Los pacientes sin complicaciones recuperaron los valores de GB, PCR y albúmina hasta llegar prácticamente a los valores preoperatorios hacia el $5^{\circ}$ día postoperatorio pero el DD permaneció elevado. En los pacientes con complicaciones la PCR y el DD continuaron aumentando sus valores después del $5^{\circ}$ día postoperatorio al mismo tiempo que la albúmina y los GB mostraban alguna recuperación. Esto se explica por la marginalización hacia la pared capilar de los GB después del alza inicial de los valores séricos. Respecto a la albúmina en los pacientes complicados, esta mostró cierta recuperación debido al soporte nutricional iniciado en estos casos, siendo esta recuperación inferior, en todo caso, a la mostrada por los pacientes sin complicaciones hacia el mismo día del postoperatorio. La recuperación de los valores normales del DD es mucho más lenta debido a que requiere un período más prolongado para alcanzar sus valores normales, esto explica porqué los valores del DD permanecieron elevados en los pacientes con y sin complicaciones.

\section{Comparación entre gastrectomía total y parcial}

Los valores preoperatorios así como los postoperatorios fueron similares en los pacientes sin complicaciones en el control preoperatorio y en los controles a las $24 \mathrm{~h}, 48 \mathrm{~h}$ y 5 días entre los pacientes sometidos a gastrectomía total y parcial. Este hecho, muestra que la injuria causada en el organismo por la remoción total o parcial del estómago fue 
similar en los pacientes del presente estudio. Aunque se podría argumentar que una resección más extensa como es la gastrectomía total tendría que producir una mayor respuesta inflamatoria que la gastrectomía parcial, debe recordarse también, que si bien una gastrectomía parcial es menos extensa también es técnicamente más demandante que una gastrectomía total y por lo tanto, relativamente más compleja, razón por la cual la respuesta inflamatoria causada por ambas técnicas es similar. La mayor o menor complejidad de la gastrectomía en pacientes con cáncer gástrico está dada por la extensión y diseminación del tumor, por esta razón y para minimizar el sesgo en las mediciones de este estudio es que la muestra está constituida por pacientes con cáncer gástrico en estadio II, los que tienen tumores relativamente pequeños, de fácil resección y sin diseminación a distancia por lo que las gastrectomías realizadas en todos estos pacientes fueron de una complejidad similar.

\section{Conclusiones}

La respuesta inflamatoria sistémica esperable en pacientes sometidos a gastrectomía total o parcial por cáncer gástrico etapa II es moderada y similar en ambas técnicas. Por esta razón la elevación marcada, precoz y sostenida de los parámetros inflamatorios habitualmente controlados en el período postoperatorio de estos pacientes, constituye un factor que contribuye a la sospecha de complicaciones después de una gastrectomía.

\section{Referencias}

1. Yamashita H, Katai H. Systemic inflammatory response in gastric cancer. World J Surg 2010;34:2399-400.

2. Crumley AB, Stuart RC, McKernan M, McMillan DC. Is hypoalbuminemia an independent prognostic factor in patients with gastric cancer? World J Surg 2010;34:2393-8.

3. Crumley AB, McMillan DC, McKernan M, Going JJ, Shearer CJ, Stuart RC. An elevated C-reactive protein concentration, prior to surgery, predicts poor cancer-specific survival in patients undergoing resection for gastroesophageal cancer. British J Cancer 2006;94:1568-71.
4. Crumley AB, McMillan DC, McKernan AC, McDonald $\mathrm{AC}$, Stuart RC. Evaluation of an inflammation-based prognostic score in patients with inoperable gastro-esophageal cancer. British J Cancer 2006;94:637-41.

5. Tas F, Ciftci R, Kilic L, Serilmez M, Karabulut S, Duranyildiz D. Clinical and prognostic significance of coagulation assays in gastric cancer. J Gastrointest Cancer 2013;44:285-92.

6. Kwon HC, Oh SY, Lee S, Kim SH, Han JY, Koh RY, et al. Plasma levels of prothrombin fragment F1+2, D-dimer and prothrombin time correlate with clinical stage and lymph node metastasis in operable gastric cancer patients. Jpn J Clin Oncol 2008;38:2-7.

7. Aliustaoglu M, Yumuk PF, Gumus M, Ekenel M, Bolukbas F, Bolukbas C, et al. D-dimer-Can it be a marker for malignant gastric lessions? Acta Oncologica 2004;43:770-1.

8. Ay C, Dunkler D, Pirker R, Thaler, Quehenberger P, Wagner $\mathrm{O}$, et al. High D-dimer levels are associated with poor prognosis in cancer patients. Haematologica 2012;97:1158-64.

9. Warschkow R, Tarantino I, Ukegjini K, Beutner U, Müller SA, Schmied BM, et al. Diagnostic study and meta-analysis of $\mathrm{C}$-reactive protein as a predictor of postoperative inflammatory complications after gastroesophageal cancer surgery. Langenbecks Arch Surg 2012;397:727-36.

10. Dutta S, Horgan PG, McMillan DC. POSSUM and its related models as predictor of postoperative mortality and morbidity in patients undergoing surgery for gastro-esophageal cancer: A systematic review. World J Surg 2010;34:2076-82.

11. Japanese Gastric Cancer Association. Japanese gastric cancer treatment guidelines 2010. Gastric Cancer 2011;14:113-23.

12. Forrest LM, McMillan DC, McArdle CS, Angerson WJ, Dunlop DJ. Evaluation of cumulative prognostic scores based on the systemic inflammatory response in patients with inoperable non-small-cell lung cancer. Br J Cancer 2003;89:1028-30.

13. Forrest LM, McMillan DC, McArdle CS, Angerson WJ, Dunlop DJ. Comparison of an inflammation-based score (GPS) with performance status (ECOG) in patients receiving platinum-based chemotherapy for inoperable non-small-cell lung cancer. Br J Cancer 2004;90:17046.

14. Dindo D, Demartines N, Clavien PA. Classification of surgical complications. Ann Surg 2004;240:205-13. 